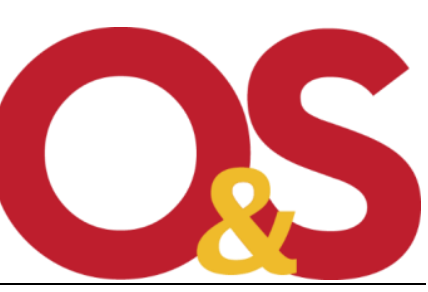

Revista Organizações \& Sociedade 2021, 28(98),458-466

(C) Autores 2021

DOI 10.1590/1984-92302021v28n9800PT

\title{
Editorial
}

\section{Impacto Político da Pesquisa}

\author{
Cintia Rodrigues de Oliveira ${ }^{a}$ \\ Eduardo Paes Barreto Davel ${ }^{b}$ \\ a Universidade Federal de Uberlândia, Uberlândia, Brasil \\ b Universidade Federal da Bahia, Salvador, Brasil
}

\section{Resumo}

Refletir sobre o impacto político abre caminhos para alargar nossa compreensão do impacto da pesquisa acadêmica e permite adensar a consciência sobre dinâmicas subjacentes e construídas socialmente, que determinam o sentido mesmo do que venha a ser coletivamente compreendido como impacto. Para pensar como os pesquisadores podem considerar plenamente o impacto político de suas pesquisas, é possível elencar, no mínimo, três grandes tipos de impacto: (a) promoção da emancipação e do pensamento crítico, (b) provocação de debate sobre o interesse público e (c) contribuição dos resultados da pesquisa para o processo de desnaturalizar conhecimentos tidos como neutros. Os impactos políticos da pesquisa acadêmica representam o futuro do desenvolvimento das sociedades e das organizações, pois viabilizam a vida em contexto coletivo, social e democrático. Se assim é, por que ainda não conseguimos consubstanciar, viabilizar e operacionalizar sua consideração em nossas métricas e diretrizes de pesquisa científica?

Palavras-chave: impacto; pesquisa; política; estudos críticos.

\section{Introdução}

Não restam dúvidas sobre os impactos econômicos, culturais e sociais que pesquisas acadêmicas podem gerar. No entanto, os equívocos sobre o próprio significado de impacto e suas dimensões estão no âmago do debate. Ao refletir sobre impacto da pesquisa acadêmica, pensamos imediatamente nas inovações, processos, produção econômica, melhoria nas condições de vida que influenciam decisões de financiamento e investimento público. Esse direcionamento imediato do 
pensamento pode excluir as motivações intrínsecas dos pesquisadores e aprofundar a dissimulação do caráter político do conhecimento (Chubb \& Reed, 2018; Reale et al., 2018), da produção acadêmica e, por conseguinte, de seus impactos.

Avançamos em termos de avaliação do impacto científico, social e político das ciências sociais e humanas. Entretanto, medir o impacto da pesquisa quanto ao que foi gerado para aumentar a competitividade científica do país, a criação de riqueza, a produtividade e bem-estar social ainda é um problema (Reale et al., 2018). O sistema de avaliação da pós-graduação no Brasil pretende avaliar o impacto econômico, social e cultural dos programas de pós-graduação, em suas atividades de formação e pesquisa, para as esferas da sociedade, governo e mercado. Essas diretrizes de impacto podem favorecer e priorizar determinados tipos de pesquisa, em termos de suas escolhas teóricas e epistemológicas. Em contrapartida, também podem afetar as motivações intrínsecas dos pesquisadores. Analisando o campo da pesquisa em administração, Wood Jr. e Costa (2015) ressaltam as críticas dirigidas ao sistema de avaliação da pós-graduação no Brasil. São críticas que sinalizam que os critérios dominantes de medição do impacto da produção científica estimulam o produtivismo, distorcendo a missão da ciência e não contribuindo para a qualidade da produção científica nacional (Wood Jr. \& Costa).

Em contrapartida, na área de administração, acadêmicos e pesquisadores encontram uma variedade de questões abordadas sob uma multiplicidade de perspectivas teóricas e epistemologias. Especificamente nos estudos organizacionais, a diversidade temática (por exemplo: estruturas organizacionais, comportamento organizacional, ética, gênero, poder e política) mostra que o campo tem reflexos das e nas problemáticas da sociedade, com contribuições teóricas e implicações práticas. Portanto, o conceito de impacto das pesquisas do campo da administração, dadas as suas características epistêmicas, é distinto daquele esperado em áreas como exatas, saúde e tecnologia. Com efeito, torna-se necessário, no Brasil, o aperfeiçoamento do ambiente institucional para que as pesquisas tenham melhor relevância e impacto (Wood Jr. \& Costa, 2015).

Em suma, a palavra-chave para avaliação de pesquisas é impacto. Entendemos que o impacto deve ser considerado nas nossas pesquisas, em suas variadas dimensões. Frente a essa variedade, elegeremos e enfocaremos o impacto político da pesquisa. Postulamos que o conhecimento gerado pelas pesquisas em administração está, em geral, imbricado em relações de poder postas pelas instituições (Foucault, 1999). Com efeito, refletir sobre o impacto político abre novos caminhos para alargar e sofisticar nossa compreensão de impacto da pesquisa acadêmica. Essa reflexão permite adensar a consciência sobre dinâmicas subjacentes e construídas socialmente, que determinam o sentido mesmo do que venha a ser coletivamente compreendido como impacto.

\section{Dimensão política da sociedade e das organizações}

O conhecimento é inseparável do poder (Foucault, 1999). Os regimes de verdade de uma sociedade - denominado por Foucault (2004) "política geral" de verdade de cada sociedade - são produtos de múltiplas coerções, estabelecendo regulamentações de poder (2004, p. 12). Cada sociedade, com seus mecanismos específicos, escolhe o conhecimento que funciona como verdadeiro. Assim como sanciona alguns mecanismos, cada sociedade distingue o falso do verdadeiro, valorizando quais técnicas e procedimentos produzem a verdade. 
Para Foucault (1999), o conhecimento científico é construído através de um processo complexo de racionalização, de identificação e classificação de objetos. Entretanto, o autor entende que o saber é aquele produzido pelo indivíduo, em um processo iterativo, sutil e vulnerável a constantes mudanças. Na geração de conhecimento científico, se estabelecem as relações de poder que expressam comando e comandados: "somos forçados a produzir a verdade pelo poder que exige essa verdade e que necessita dela para funcionar, temos de dizer a verdade, somos coagidos, somos condenados a confessar a verdade ou encontrá-la" (1999, p. 29).

Como sugere Mitre (2016), será que a relação entre ciência e política é paradoxal? Por um lado, a ciência ganha legitimidade por ser capaz de orientar a elaboração e execução de políticas públicas. Por outro, pode ter sua legitimidade ameaçada pela vinculação com argumentos defendidos no campo político (Gieryn, 1983). Nesse contexto, interesses de grupos são colocados em disputa, vislumbrando-se a ideia de que a ciência é socialmente construída, visto que interesses e ideologias trazem implicações para a aceitação ou rejeição de postulados científicos (Brown, 2009). A sociologia do conhecimento científico argumentou que fatores sociais poderiam trazer melhor compreensão sobre os fatos científicos (Bloor, 1991), sustentando que a realidade é socialmente construída (Berger \& Luckmann, 1966).

Na construção social da ciência, subtende-se que a política é uma dimensão central na construção da pesquisa acadêmica (Latour, 1987; Latour \& Woolgar, 1986). Como o conhecimento é político, a tentativa de separar ciência e política em função de uma suposta objetividade científica não elimina dos fenômenos estudados a totalidade social da qual estão revestidos. No campo da administração, no qual as relações entre organizações e sociedade são centrais, a pesquisa que objetiva a generalização do conhecimento e a neutralidade científica (próprias das ciências naturais) indica uma pretensão ou uma perigosa cilada. A consideração do contexto, dos valores, do poder e dos interesses específicos é capaz de gerar resultados de pesquisa mais conscientes, mais refletidos, que considerem uma perspectiva sócio-histórica-temporal mais abrangente. É capaz, inclusive, de gerar de forma inovadora perspectivas que expliquem melhor o fenômeno estudado, conduzindo a uma ação mais profícua para transformar a realidade (Clegg \& Palmer, 1996; Flyvbjerg, 2006).

As organizações e a administração são construções sociais e políticas. Como tais, resultam das interações entre os atores envolvidos, que as interpretam ao mesmo tempo que as influenciam. A produção científica sobre organizações e administração gera conhecimento com impacto político se provocar a transformação da realidade estudada. Por isso, as pesquisas precisam melhor envolver os participantes nativos do campo empírico para potencializar a geração de resultados úteis para as políticas e as práticas do contexto estudado (Misoczky, Flores, \& Moraes, 2010; Paes de Paula, 2007).

Uma das questões centrais no impacto político da pesquisa em administração está atrelada à relação entre a sociedade e empresas orientadas pelo lucro. Essa relação é marcada pelo protagonismo das empresas nas transformações sociais, tecnológicas e econômicas que impactam comportamentos de consumo, estilos de vida e éticas de trabalho, ao mesmo tempo que restringem o acesso a recursos naturais. Essas relações revelam-se paradoxais frente ao cenário de desequilíbrio em que a sociedade contemporânea se encontra. Por exemplo, enfrentamos desequilíbrios constantes entre o avanço tecnológico e os níveis de pobreza e desigualdade econômica e social, entre a melhoria dos índices de produtividade e o respeito aos direitos humanos, entre a sustentabilidade ambiental e a responsabilidade ambiental, o que perpetua a 
dinâmica de desigualdades (Dyer, Humphries, Fitzgibbons, \& Hurd, 2014; O’Neil, 2016; Zuboff, 1988, 2019). Como repensar os resultados da pesquisa e permeá-los de preocupações com seus potenciais impactos políticos? Como as pesquisas poderiam integrar uma preocupação mais nítida e robusta sobre os impactos políticos acarretados pelos seus resultados?

As pesquisas na área de administração podem contribuir para uma melhor compreensão sobre como os impactos de seus resultados serão negativos e/ou positivos para organizações, humanos, comunidades, animais, sociabilidades, natureza, fazeres, saberes etc. Os resultados podem, inclusive, ajudar na identificação e reflexão sobre práticas não éticas, condutas criminosas e a suposta aura pura de algumas teorias organizacionais. É isso que o movimento denominado estudos críticos em administração estimula: a produção de conhecimento a partir de reflexões críticas e questionamentos de verdades absolutas (Alvesson \& Deetz, 2000; Alvesson, Bridgman, \& Willmott, 2009; Davel \& Alcadipani, 2003), de modo que reflita as dinâmicas locais (Abdalla \& Faria, 2017) e nativas. Com efeito, a geração de conhecimento sobre organizações e administração, que toma os problemas locais e nativos como referências e que reconhece a dimensão política da epistemologia (Misoczky \& Camara, 2020), torna-se imprescindível para estimular a reflexão crítica e emancipadora sobre os impactos da pesquisa acadêmica.

\section{Impacto político da pesquisa acadêmica}

Poder, política e influência são temas pesquisados, há muito tempo, no campo da administração (e. g. Clegg, 1975, 1979; Clegg, \& Palmer, 1996; Clegg, Courpasson, \& Phillips, 2006; Faria, 2007, 2017; Fleming \& Spicer, 2014; Frost, 1987; Goss, Jones, Betta, \& Latham, 2011; Hardy, 1995; Hardy \& Clegg, 2006; Jermier, Knights, \& Nord, 1994; McKinlay \& Starkey, 1998; Mumby, 1988; Townley, 1993), revendo as dimensões mais diretas ou subterrâneas, estruturais ou processuais na prática de gestores, líderes, empreendedores e profissionais nas organizações. Esses temas constituem as principais inquietações e motivações dos estudos críticos em administração (e.g. Adler, 2002, 2007; Adler, Forbes, \& Willmott, 2007; Alvesson, 2011; Alvesson \& Willmott, 1992, 2003; Alvesson et al., 2009; Davel \& Alcadipani, 2003; Fournier \& Grey, 2000; Maranhão \& Vilela, 2017; Paes de Paula, 2007; Smircich \& Calàs, 1995), que já produziram conhecimento acadêmico que ajuda a orientar as pesquisas em termos metodológicos (e. g. Alvesson \& Ashcraft, 2009; Alvesson \& Deetz, 2000; Alvesson \& Sköldberg, 2000) e educacionais (e. g. Abdala \& Faria, 2017; Grey, 1996, 2004; Misoczky \& Camara, 2020). Mesmo com essa farta produção acadêmica, como podemos melhor entender o impacto político da pesquisa acadêmica? Como mensurar tal impacto? O impacto - entendido muitas vezes como mudanças provenientes dos resultados gerados pelas pesquisas acadêmicas - apresenta desafios e problemas para ser avaliado, a exemplo dos dispositivos e métodos de medição. Como podemos assegurar que tais resultados foram as causas mais significativas das mudanças geradas (Reale et al., 2018)? Em relação ao impacto político, a avalição não se faz com métricas.

Para pensar de que forma pesquisadores podem considerar plenamente o impacto político de suas produções, podemos pensar em, no mínimo, três grandes tipos de impacto. O primeiro refere-se ao impacto como promoção da emancipação e do pensamento crítico. Os resultados de pesquisas podem contribuir para desenvolver o pensamento crítico das pessoas e promover níveis progressivos de emancipação. Pesquisadores e pesquisados desafiam verdades enraizadas nas 
estruturas organizacionais e formas de pensamento vigentes, levando a mudanças políticas fundamentais que podem melhorar as relações e condições locais.

Esse tipo de impacto pode ser facilmente originado de pesquisas que encorajem os participantes a se conscientizarem de sua realidade e de sua capacidade de transformá-la, de fazer a diferença no contexto em que atua. O pensamento crítico é importante para a vida das pessoas, assim, precisa ser estimulado e fortalecido por meio de um exercício reflexivo permanente. Grupos específicos, como mulheres, trabalhadores, membros de comunidades e, também, os pesquisadores, se descobrem capazes de tomar decisões a partir de experimentações, pois pensar criticamente implica a junção do conhecimento e da disposição para agir com base na reflexão. Para isso, as pesquisas têm a oportunidade de potencializar a quebra de preconceitos e estereótipos ao invés de reforçá-los. Podem fomentar conhecimentos em que as diferenças humanas sejam não só reconhecidas, mas compreendidas e valorizadas.

O segundo tipo de impacto consiste na provocação de debate sobre o interesse público. Os resultados de pesquisas podem provocar debates sobre assuntos até então não percebidos como problemáticos em relação ao interesse coletivo. Ao disseminar suas reflexões para além da audiência acadêmica, pesquisadores demonstram um esforço para explicar que a pesquisa faz parte de um projeto político mais amplo. Essa preocupação com a alimentação do debate coletivo, acadêmico e extra-acadêmico, revela os impactos políticos que um resultado de pesquisa pode provocar. Além de repensar a divulgação dos resultados, pesquisadores devem "ouvir" o público e ampliar as formas de participação social, considerando que os sujeitos da pesquisa são também os interessados na aplicação dos resultados. Pesquisas que considerem a relação profunda e profícua entre o conhecimento científico e a sociedade são capazes de considerar plenamente e revelar a teia de relações de poder, as disputas, as resistências e negociações que envolvem uma pesquisa acadêmica.

O terceiro tipo de impacto refere-se à contribuição que os resultados da pesquisa oferecem para o processo de desnaturalizar conhecimentos tidos como neutros. Desnaturalizar pode ser entendido como um processo contínuo de estranhar o que se sabe e colocar em suspensão para a prática reflexiva, a interrogação, o questionamento, e assim reorganizar o que se conhece. Organizações são edificadas como lugares de racionalidade, porém, ao contrário, são espaços não neutros na re-desconstrução de identidades, símbolos, discursos, lugares e modelos. Os resultados de pesquisas que confrontem, questionem e estranhem modelos e saberes podem dar visibilidade a questões e entendimentos até então percebidos como normais, naturais e neutros. A pesquisa e seus resultados devem ser compreendidos como um ato político. Se pesquisas tendem a legitimar modelos, concepções e ferramentas, então, que modelos, concepções e ferramentas o(a) pesquisador(a) deseja legitimar? Quem se beneficia dessa legitimação?

Os impactos políticos são substantivos e ultrapassam métricas lineares e objetivas. Além disso, são potencializados tanto durante quanto após o processo da pesquisa, ou seja, não somente nos resultados, como também no modo como os pesquisadores realizam suas pesquisas, nas interações com o campo empírico e no modo como os formuladores de políticas públicas comunicam suas decisões (Reale et al., 2018). Isso significa que pesquisadores(as) precisam se preocupar em promover a participação de todas as pessoas e organizações envolvidas na pesquisa, com a avaliação de seus mais diversos tipos de impactos. 
Os impactos políticos da pesquisa acadêmica representam o futuro do desenvolvimento das sociedades e das organizações, na medida que viabilizam a vida em contexto coletivo, social e democrático. Se assim é, por que ainda não conseguimos consubstanciar, viabilizar e operacionalizar sua consideração em nossas métricas e diretrizes de pesquisa científica?

\section{Referências}

Abdalla, M., \& Faria, A. (2017). Em defesa da opção decolonial em administração/gestão. Cadernos EBAPE.BR, 15(4), 1-16. doi:10.1590/1679-395155249

Adler, P. (2002). Critical in the name of whom and what?. Organization, 9(3), 387-395. doi:10.1177\%2F135050840293003

Adler, P. (2007). The future of critical management studies: A paleo-marxist critique of labour process theory. Organization Studies, 28(9), 1313-1345. doi:10.1177/0170840607080743

Adler, P., Forbes, L. C., \& Willmott, H. (2007). Critical management studies. Academy of Management Annals, 1(1), 119-179. doi:10.1080/078559808

Alvesson, M. (2011). Classics in critical management studies. Cheltenham: Edward Elgar.

Alvesson, M., \& Ashcraft, K. L. (2009). Critical methodology in management and organization research. In D. A. Buchanan, \& A. Bryman (Eds.), The SAGE Handbook of organizational research methods (pp. 61-77). Los Angeles: Sage.

Alvesson, M., \& Deetz, S. (2000). Doing critical management research. Londres: Sage.

Alvesson, M., \& Sköldberg, K. (2000). Reflexive methodology: new vistas for qualitative research. Londres: Sage.

Alvesson, M., \& Willmott, H. (1992). Critical management studies. Londres: Sage.

Alvesson, M., \& Willmott, H. (2003). Studying management critically. Londres: Sage.

Alvesson, M., Bridgman, T., \& Willmott, H. (2009). The Oxford handbook of critical management studies. Oxford: Oxford University Press.

Berger, P. L., \& Luckmann, T. (1966). The Social Construction of Reality. Harmondsworth: Penguin.

Bloor, D. (1991). Knowledge and social imagery. Chicago: University of Chicago Press.

Brown, M. B. (2009). Science in democracy. Expertise, institutions and representation. Cambridge: The MIT Press.

Chubb, J., \& Reed, M. S. (2018). The politics of research impact: academic perceptions of the implications for research funding, motivation and quality. British Politics, 13(3), 295-311. doi:10.1057/s41293-018-0077-9

Clegg, S. (1975). Power, rule, and domination: a critical and empirical understanding of power in sociological theory and organizational life. Londres: Routledge.

Clegg, S. (1979). The theory of power and organization. Londres: Routledge.

Clegg, S., \& Palmer, G. (1996). The politics of management knowledge. Londres: Sage. 
Clegg, S., Courpasson, D., \& Phillips, N. (2006). Power and organizations. Londres: Sage.

Davel, E., \& Alcadipani, R. (2003). Estudos críticos em administração: a produção científica brasileira nos anos 1990. Revista de Administração de Empresas, 43(4), 72-85. doi:10.1590/S0034-75902003000400006

Dyer, S., Humphries, M., Fitzgibbons, D., \& Hurd, F. (2014). Understanding management critically. Londres: Sage.

Faria, J. H. (2007). Análise Crítica das Teorias e Práticas Organizacionais. São Paulo, SP: Atlas.

Faria, J. H. (2017). Poder, Controle e Gestão. Curitiba, PR: Juruá.

Fleming, P., \& Spicer, A. (2014). Power in management and organization science. Academy of Management Annals, 8(1), 237-298. doi:10.5465/19416520.2014.875671

Flyvbjerg, B. (2006). Making organization research matter: power, values and phronesis. In S. R. Clegg, C. Hardy, T. B. Lawrence, \& W. R. Nord (Eds.), The SAGE Handbook of organization studies (pp. 370-387). Londres: Sage.

Foucault, M. (1999). Em defesa da sociedade: curso no College de France (1975-1976). São Paulo, SP: Martins Fontes.

Foucault, M. (2004). Microfísica do poder. 23a ed. São Paulo, SP: Graal.

Fournier, V., \& Grey, C. (2000). At the critical moment: conditions and prospects for critical management studies. Human Relations, 53(1), 7-32. doi:10.1177/0018726700531002

Frost, P. J. (1987). Power, politics, and influence. In F. Jablin, L. Putnam, K. H. Roberts, \& L. W. Porter (Eds.), Handbook of organizational communication: an interdisciplinary perspective (pp. 586-624) Newbury Park: Sage.

Gieryn, T. (1983). Boundary-work and the Demarcation of Science from Non-sciences: Strains and Interests in Professional Ideologies of Scientists. American Sociological Review, 48(6), 781-795. doi:10.2307/2095325

Goss, D., Jones, R., Betta, M., \& Latham, J. (2011). Power as practice: A micro-sociological analysis of the dynamics of emancipatory entrepreneurship. Organization Studies, 32(2), 211-229. doi:10.1177/0170840610397471

Grey, C. (1996). Critique and renewal in management education. Management Learning, 27(1), 5-20.

Grey, C. (2004). Reinventing Business Schools: The Contribution of Critical Management Education. Academy of Management Learning \& Education, 3(2), 178-196. doi:10.5465/amle.2004.13500519

Hardy, C. (Ed.). (1995). Power and Politics in Organizations. Aldershot: Dartmouth.

Hardy, C., \& Clegg, S. (2006). Some dare call it power. In S. R. Clegg, C. Hardy, T. B. Lawrence, \& W. R. Nord (Eds.), The SAGE Handbook of organization studies (pp. 754-775). Londres: Sage.

Jermier, J. M., Knights, D., \& Nord, W. (1994). Resistance and power in organizations. Londres: Routledge. 
Latour, B. (1987). Science in action: how to follow scientists and engineers through society. Cambridge: Harvard University Press.

Latour, B., \& Woolgar, S. (1986). Laboratory life: the construction of scientific facts (2a ed.). Princeton: Princeton University Press.

Maranhão, C. S., \& Vilela, J. R. P. X. (2017). A imanência entre a teoria crítica e a pesquisa empírica: contribuições para os estudos organizacionais. Organizações \& Sociedade, 24(82), 476-490. doi:10.1590/1984-9240826

McKinlay, A., \& Starkey, K. (1998). Foucault, management and organization theory: from panopticon to technologies of self. Londres: Sage.

Misoczky, M. C. A., \& Camara, G. D. (2020). Pensar desde a América Latina em diálogo com a organização das lutas sociais descoloniais: Explorando possibilidades. RAE-Revista de Administração de Empresas, 60(2), 93-103. doi:10.1590/S0034-759020200203

Misoczky, M. C., Flores, R. K., \& Moraes, J. (2010). Organização e Práxis Libertadora. Porto Alegre, RS: Dacasa.

Mitre, M. (2016). As relações entre ciência e política, especialização e democracia: a trajetória de um debate em aberto. Estudos Avançados, 30(87), 279-298. doi: 10.1590/S010340142016.30870016

Mumby, D. K. (1988). Communication and power in organizations: discourse, ideology, and domination. Norwood: Ablex.

O'Neil, C. (2016). Weapons of math destruction: How big data increases inequality and threatens democracy. Nova lorque: Crown.

Paes de Paula, A. P. (2007). Teoria crítica nas organizações. São Paulo, SP: Thomson Pioneira.

Reale, E., Avramov, D., Canhial, K., Donovan, C., Flecha, R., Holm, P., . . Van Horik, R. (2018). A review of literature on evaluating the scientific, social and political impact of social sciences and humanities research, Research Evaluation, 27(4), 298-308. doi:10.1093/reseval/rvx025

Smircich, L., \& Calàs, M. (1995). Critical perspectives on organization and management. Aldershot: Dartmouth.

Townley, B. (1993). Foucault, power/knowledge, and its relevance for human resource management. Academy of Management Review, 18(3), 518-545. doi:10.2307/258907

Wood Jr, T., \& Costa, C. C. M. (2015). Avaliação do impacto da produção científica de programas selecionados de pós-graduação em Administração por meio do índice $\mathrm{H}$. Revista de Administração RAUSP, 50(3), 325-337. doi: 10.5700/rausp1203

Zuboff, S. (1988). In the age of the smart machine: the future of work and power. Nova lorque: Basic.

Zuboff, S. (2019). The age of surveillance capitalism: The fight for a human future at the new frontier of power. Londres: Profile Books. 


\section{Autoria}

\section{Cintia Rodrigues de Oliveira}

Doutora com pós-doutorado em administração pela Escola de Administração de Empresas de São Paulo, da Fundação Getulio Vargas. Professora na Faculdade de Gestão e Negócios da Universidade Federal de Uberlândia. Professora visitante da Faculty of Business and Law da Open University (Reino Unido). Editora associada da revista Organizações \& Sociedade para o eixo "Organizações, Política e Sociedade". Pesquisa e publica sobre cultura, sociedade e poder, crimes corporativos e o dark side das organizações.

E-mail: cintia@ufu.br

ORCID: https://orcid.org/0000-0001-7999-9002

\section{Eduardo Paes Barreto Davel}

Doutor em administração pela École des Hautes Études Commerciales de Montréal (Canadá), com pós-doutorado em administração pela Nova School of Business and Economics da Universidade Nova de Lisboa (Portugal). Professor na Escola de Administração da Universidade Federal da Bahia. Editor-chefe da revista Organizações \& Sociedade. Pesquisa e publica sobre empreendedorismo cultural, gestão do processo criativo, ensino, metodologia, aprendizagem, cultura e estética nas organizações.

E-mail: davel.eduardo@gmail.com

ORCID: https://orcid.org/0000-0003-0610-6474

\section{Contribuição dos autores}

Primeira autora: concepção (igual), investigação (igual), redação - rascunho original (igual).

Segundo autor: concepção (igual), investigação (igual), redação - rascunho original (igual).

A O\&S é signatária do DORA (The Declaration on Research Assessment) e do COPE (Committee on Publication Ethics).

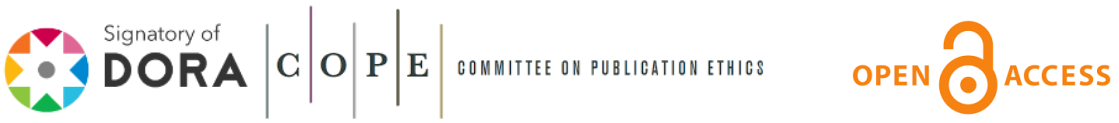

